Jianlian Liu*, Hong Hao and Chaojun Du

\title{
Crystal structure of $(E)-N^{\prime}-(2-c h l o r o-$ 6-hydroxybenzylidene)- 2-hydroxybenzohydrazide, $\mathrm{C}_{14} \mathrm{H}_{11} \mathrm{ClN}_{2} \mathrm{O}_{3}$
}

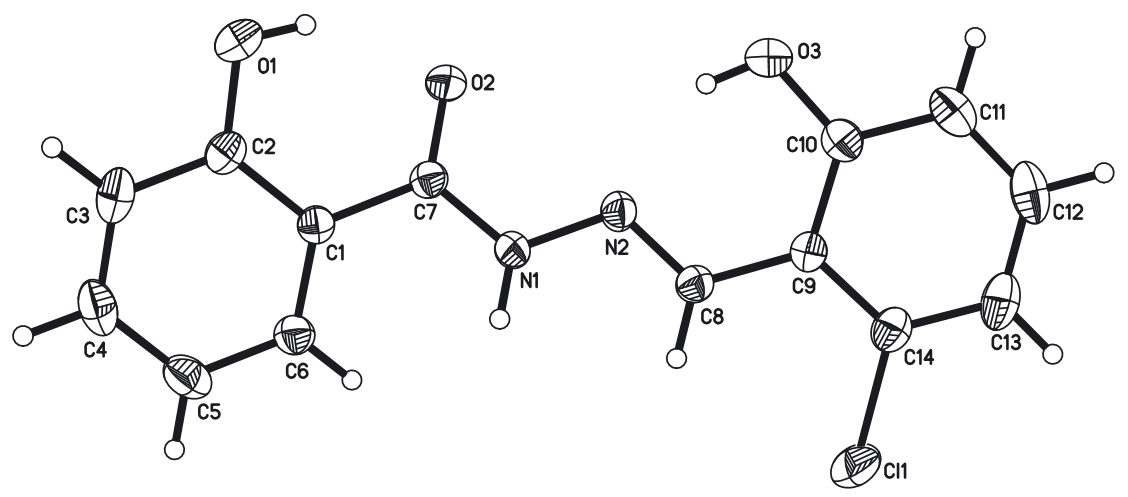

https://doi.org/10.1515/ncrs-2021-0154 Received April 23, 2021; accepted May 10, 2021; published online June 18, 2021

\begin{abstract}
$\mathrm{C}_{14} \mathrm{H}_{11} \mathrm{ClN}_{2} \mathrm{O}_{3}$, monoclinic, $P 2_{1} / n$ (no. 14), $a=7.0242(11) \AA$, $b=25.375(4) \AA, c=7.6789(13) \AA, \quad \beta=111.2752^{\circ}$, $V=1275.4(4) \AA^{3}, Z=4, R_{g t}(F)=0.0349, w R_{r e f}\left(F^{2}\right)=0.1123$, $\mathrm{T}=296(2) \mathrm{K}$.
\end{abstract}

\section{CCDC no.: 2082667}

The asymmetric unit of the title structure is shown in the figure. Table 1 contains crystallographic data and Table 2 contains the list of the atoms including atomic coordinates and displacement parameters.

*Corresponding author: Jianlian Liu, School of Chemical Engineering, Northwest University, 710069, Xi'an, Shaanxi, People's Republic of China; and School of Biological and Chemical Engineering, Nanyang Institute of Technology, 473004, Nanyang, Henan, People's Republic of China, E-mail: zgqjd2005@sohu.com. https://orcid.org/00000001-9270-2293

Hong Hao, School of Chemical Engineering, Northwest University, 710069, Xi'an, Shaanxi, People's Republic of China,

E-mail: tianxtpljh2002@163.com

Chaojun Du, School of Biological and Chemical Engineering, Nanyang Institute of Technology, 473004, Nanyang, Henan, People's Republic of China

Ә Open Access. ( 2021 Jianlian Liu et al., published by De Gruyter. ((c))BY License.
Table 1: Data collection and handling.

\begin{tabular}{ll}
\hline Crystal: & Colourless block \\
Size: & $0.25 \times 0.23 \times 0.20 \mathrm{~mm}$ \\
Wavelength: & Mo $K \alpha$ radiation $(0.71073 \AA$ A \\
$\mu:$ & $0.31 \mathrm{~mm}^{-1}$ \\
Diffractometer, scan mode: & Bruker APEX-II, $\varphi$ and $\omega$ \\
$\theta_{\text {max }}$, completeness: & $25.0^{\circ},>99 \%$ \\
$N(h k l)_{\text {measured }}, N(h k l)_{\text {unique }}, R_{\text {int }}:$ & $6399,2245,0.023$ \\
Criterion for $l_{\text {obs }}, N(h k l)_{\text {gt: }}:$ & $I_{\text {obs }}>2 \sigma\left(I_{\text {obs }}\right), 1909$ \\
$N(\text { param })_{\text {refined }}:$ & 183 \\
Programs: & Bruker [1], SHELX [2] \\
\hline
\end{tabular}

\section{Source of material}

A mixture of 2-hydroxy-benzoic acid hydrazide $(152.2 \mathrm{mg}$, $1 \mathrm{mmol}$ ) and 2-chloro-6-hydroxybenzaldehyde (156.6 mg, $1 \mathrm{mmol}$ ) in $50 \mathrm{~mL}$ anhydrous ethanol containing a few drops of glacial acetic acid was stirred under reflux conditions for $2 \mathrm{~h}$. The mixture was cooled to room temperature and filtered, and then left at room temperature. After a few days, yellow block-shaped crystals suitable were obtained.

\section{Experimental details}

The hydrogen atoms were positioned geometrically $(\mathrm{C}-\mathrm{H}=0.93 \AA, \mathrm{N}-\mathrm{H}=0.86 \AA, \mathrm{O}-\mathrm{H}=0.82 \AA)$ and refined as riding atoms. The $U_{\text {iso }}$ values were constrained to be $1.2 U_{\mathrm{eq}}$ of the carrier atom for carbon bound $\mathrm{H}$ atoms and $1.5 U_{\text {eq }}$ for the remaining $\mathrm{H}$ atoms. 
Table 2: Fractional atomic coordinates and isotropic or equivalent isotropic displacement parameters $\left(\AA^{2}\right)$.

\begin{tabular}{lrrrr}
\hline Atom & $\boldsymbol{x}$ & $\boldsymbol{y}$ & $\boldsymbol{z}$ & $\boldsymbol{U}_{\text {iso* }} \boldsymbol{U}_{\text {eq }}$ \\
\hline C1 & $0.6707(2)$ & $0.32492(6)$ & $0.6496(2)$ & $0.0264(4)$ \\
C2 & $0.6835(2)$ & $0.37973(6)$ & $0.6306(2)$ & $0.0311(4)$ \\
C3 & $0.7307(3)$ & $0.41154(7)$ & $0.7874(3)$ & $0.0418(4)$ \\
H3 & 0.743319 & 0.447752 & 0.776416 & $0.050^{*}$ \\
C4 & $0.7590(3)$ & $0.38989(8)$ & $0.9589(3)$ & $0.0453(5)$ \\
H4 & 0.790634 & 0.411732 & 1.062837 & $0.054^{*}$ \\
C5 & $0.7414(3)$ & $0.33620(8)$ & $0.9798(2)$ & $0.0423(4)$ \\
H5 & 0.758484 & 0.321968 & 1.096153 & $0.051^{*}$ \\
C6 & $0.6979(2)$ & $0.30405(6)$ & $0.8250(2)$ & $0.0328(4)$ \\
H6 & 0.686594 & 0.267871 & 0.837962 & $0.039^{*}$ \\
C7 & $0.6280(2)$ & $0.29130(6)$ & $0.4830(2)$ & $0.0272(3)$ \\
C8 & $0.7219(2)$ & $0.16077(6)$ & $0.4004(2)$ & $0.0290(4)$ \\
H8 & 0.767364 & 0.149250 & 0.523688 & $0.035^{*}$ \\
C9 & $0.7070(2)$ & $0.12389(6)$ & $0.2529(2)$ & $0.0272(3)$ \\
C10 & $0.6617(2)$ & $0.14042(6)$ & $0.0664(2)$ & $0.0328(4)$ \\
C11 & $0.6581(3)$ & $0.10471(8)$ & $-0.0710(3)$ & $0.0442(5)$ \\
H11 & 0.631030 & 0.116142 & -0.192701 & $0.053^{*}$ \\
C12 & $0.6948(3)$ & $0.05238(8)$ & $-0.0267(3)$ & $0.0513(5)$ \\
H12 & 0.691889 & 0.028650 & -0.119913 & $0.062^{*}$ \\
C13 & $0.7357(3)$ & $0.03403(7)$ & $0.1519(3)$ & $0.0462(5)$ \\
H13 & 0.757917 & -0.001654 & 0.179249 & $0.055^{*}$ \\
C14 & $0.7429(2)$ & $0.06981(6)$ & $0.2892(2)$ & $0.0351(4)$ \\
C11 & $0.79733(9)$ & $0.04664(2)$ & $0.51507(7)$ & $0.0589(2)$ \\
N1 & $0.69976(19)$ & $0.24167(5)$ & $0.51329(18)$ & $0.0310(3)$ \\
H1A & 0.761624 & 0.230390 & 0.625330 & $0.037^{*}$ \\
N2 & $0.6730(2)$ & $0.20921(5)$ & $0.36294(18)$ & $0.0300(3)$ \\
01 & $0.6557(2)$ & $0.40315(5)$ & $0.46567(18)$ & $0.0452(3)$ \\
H1 & 0.618765 & 0.381112 & 0.381920 & $0.068^{*}$ \\
02 & $0.53252(19)$ & $0.30788(4)$ & $0.32239(16)$ & $0.0380(3)$ \\
O3 & $0.6208(2)$ & $0.19117(5)$ & $0.01546(18)$ & $0.0471(4)$ \\
H3A & 0.631904 & 0.209004 & 0.107700 & $0.071^{*}$ \\
\hline & & & &
\end{tabular}

\section{Comment}

Halogenated Schiff bases are attracting more and more attention in several fields such as biochemistry, pharmacy, and coordination chemistry [3]. In addition, halogenated compounds containing the hydrazide moiety form intramolecular H-bonds [4-11]. In order to search for new Schiff bases containing halogen atoms, the title compound was synthesized and its crystal structure is reported here.

The asymmetric unit of the title compound consists of one formula unit ( $c f$. the figure). The molecule exhibits an $E$ configuration. The bond length of $\mathrm{C} 8=\mathrm{N} 2$ is $1.2804(19) \AA$ exhibiting that the $\mathrm{C} 8=\mathrm{N} 2$ bond is a double bond. The two aromatic rings form the dihedral angle of $14.3^{\circ}$. The angle of $\mathrm{C} 8=\mathrm{N} 2-\mathrm{N} 1$ is $116.29(13)^{\circ}$, smaller than the ideal value of $120^{\circ}$.
In the crystal packing, the chain structure was formed by intermolecular $\mathrm{N}-\mathrm{H} \cdots \mathrm{O}$ hydrogen bonds. The intramolecular $\mathrm{O}-\mathrm{H} \cdots \mathrm{O}$ and $\mathrm{N}-\mathrm{H} \cdots \mathrm{O}$ hydrogen bonds also exist, which further consolidate the crystal.

Author contributions: All the authors have accepted responsibility for the entire content of this submitted manuscript and approved submission.

Research funding: Education Department of Henan Province (20B530004), Nanyang Science and Technology Bureau (JCQY004), and Nanyang Key Laboratory of Catalytic Functional Materials.

Conflict of interest statement: The authors declare no conflicts of interest regarding this article.

\section{References}

1. Bruker. APEX2, SAINT and SADABS; Bruker AXS Inc.: Madison, Wisconsin, USA, 2008.

2. Sheldrick G. M. A short history of SHELX. Acta Crystallogr. 2008, A64, 112-122.

3. Wu Q., Huang M., Li T., Jiao L., Tu Y., Xu X., Ma X., Tian H., Qiao Y. Crystal and electronic structure of poly-halogenated lanthanide Schiff base complex: insights into halogen bond from structural and theoretical analysis. J. Mol. Struct. 2021, 1225, 129054.

4. Li W., Yang B. Crystal structure of 2,2'-[ethane-1,2diylbis(azanylylidenemethylylidene)]bis(6-chlorophenol), $\mathrm{C}_{16} \mathrm{H}_{14} \mathrm{C}_{12} \mathrm{~N}_{2} \mathrm{O}_{2}$. Z. Kristallogr. N. Cryst. Struct. 2021, 236, 599-600.

5. Xu X., Ma X., Huang M., Li T., Wu Q. Crystal structure of 4-bromo(N)'-[(3-bromo-2-hydroxyphenyl)methylidene]benzohydrazide methanol solvate, $\mathrm{C}_{15} \mathrm{H}_{14} \mathrm{Br}_{2} \mathrm{~N}_{2} \mathrm{O}_{3}$. Z. Kristallogr. N. Cryst. Struct. 2021, 236, 323-324.

6. Chen J., Liu Z., Yan H. Crystal structure of (E)-(N)'-(2- chloro6-hydroxy-benzylidene)-4-hydroxybenzohydrazidedihydrofuran- $2(3 \mathrm{H})$-one (1/1), $\mathrm{C}_{18} \mathrm{H}_{17} \mathrm{ClN}_{2} \mathrm{O}_{5}$. Z. Kristallogr. $\mathrm{N}$. Cryst. Struct. 2020, 235, 997-998.

7. Yan X., Zhong S., Zhang H. The crystal structure of $(E)$ - $(N)$ '-(1(3-chloro-4-fluorophenyl) ethylidene)-2-hydroxybenzohydrazide, $\mathrm{C}_{15} \mathrm{H}_{12} \mathrm{ClFN}_{2} \mathrm{O}_{2}$. Z. Kristallogr. N. Cryst. Struct. 2020, 235, 263-265.

8. Liu W. Crystal structure of (N)', 2-bis((E)-2-chloro-6hydroxybenzylidene)hydrazine-1-carbothiohydrazide, $\mathrm{C}_{15} \mathrm{H}_{12} \mathrm{Cl}_{2} \mathrm{~N}_{4} \mathrm{O}_{2}$ S. Z. Kristallogr. N. Cryst. Struct. 2020, 235, 99-100.

9. Song W., Yang Y., Li J. Crystal structure of (E)-2-(2-chloro-6hydroxybenzylidene)hydrazinecarbothioamide, $\mathrm{C}_{8} \mathrm{H}_{8} \mathrm{CIN}_{3} \mathrm{OS}$. Z. Kristallogr. N. Cryst. Struct. 2020, 235, 61-62.

10. Song W., Yang Y. Crystal structure of $(E)$-3-chloro-2(((4-chlorophenyl)imino)methyl)phenol. Z. Kristallogr. N. Cryst. Struct. 2020, 235, 29-30.

11. Chang F.-F., Zhang L., Zhao P.-C., Huang W. Transmetalation for flexible pendant-armed Schiff-base macrocyclic complexes influenced by halide effects. Inorg. Chem. 2018, 57, 1438-1448. 